\title{
Elementos comunicacionais da estratégia das organizações'
}

\author{
Communication elements of organizational strategy
}

\section{Elementos comunicacionales de la estrategia de las organizaciones}

\section{Victor Márcio Laus Reis Gomes}

- $\quad$ Doutor em Comunicação Social pela Pontifícia Universidade Católica do Rio Grande do Sul (PUC-RS)

- Mestre em Administração pela Universidade do Vale do Rio dos Sinos (Unisinos)

- $\quad$ Master of Business Administration (MBA) pela University of Dallas

- $\quad$ Especialista em Marketing e bacharel em Comunicação Social pela PUC-RS

- $\quad$ Foi professor da Escola Superior de Propaganda e Marketing (ESPM-Sul) e da Faculdade Porto-Alegrense (Fapa)

- $\quad$ Atuou como executivo de marketing e comunicação em empresas como RBS, Claro e Dell

- $\quad$ É professor e pesquisador do Programa de Pós-Graduação em Comunicação da Universidade Católica de Brasília (UCB).

- E-mail: victorlaus@gmail.com

1 Este artigo baseia-se em nossa tese de doutorado, "Uma leitura comunicacional da estratégia na perspectiva sistêmico-discursiva", desenvolvida com o apoio da Coordenação de Aperfeiçoamento de Pessoal de Nível Superior (Capes) e defendida em 2014 no Programa de Pós-Graduação em Comunicação Social da Pontifícia Universidade Católica do Rio Grande do Sul (PUC-RS), sob a orientação da Profa. Dra. Cláudia Peixoto de Moura. Ela obteve o 3 lugar entre as teses, no Prêmio Abrapcorp de Teses e Dissertações 2015, conferido no IX Congresso Brasileiro Científico de Comunicação Organizacional e de Relações Públicas, realizado em Campinas (SP) de 13 a 15 de maio de 2015 . 


\section{Resumo}

Em uma reflexão teórica, este artigo discute um conjunto de elementos comunicacionais que podem ser aplicados para a compreensão da estratégia das organizações. Esses elementos são categorizados da seguinte forma: a decisão, a identificação, a institucionalização, a mediação, a cultura organizacional e a comunicação organizacional. A articulação desses elementos indica que as estratégias são constituídas, negociadas e representadas, por meio da processualidade da comunicação organizacional, em um conjunto de decisões voltadas para a diferenciação das organizações.

\section{PALAVRAS-CHAVE: COMUNICAÇÃO • ESTRATÉGIA •SISTEMA • DISCURSO.}

\section{Abstract}

This paper offers a theoretical reflection on a set of communicational elements that may be applied in order to understand organizational strategy. These elements are categorized as follows: decision, identification, institutionalization, mediation, organizational culture and organizational communication. The articulation of such elements shows that the strategies are formed, negotiated and represented through organizational communication processuality in a combination of decisions aimed at differentiation of the organizations.

\section{KEYWORDS: COMMUNICATION・STRATEGY •SYSTEM・DISCOURSE.}

\section{Resumen}

En una reflexión teórica, este artículo discute una serie de elementos comunicacionales que se pueden aplicar a la comprensión de la estrategia de las organizaciones. Estos elementos se clasifican de la siguiente manera: la decisión, la identificación, la institucionalización, la mediación, la cultura organizacional y la comunicación organizacional. La articulación de estos elementos indica que las estrategias son constituidas, negociadas y representadas por medio de la procesualidad de la comunicación organizacional en una serie de decisiones encaminadas a la diferenciación de las organizaciones. 
$\mathrm{E}$

m abordagens econômicas e administrativas, a estratégia das organizações é compreendida, de forma sintética, como um conjunto de decisões que: definem uma visão e uma missão sustentáveis (Ansoff, 1983; Chandler, 1998); alocam recursos e capacitam uma organização a conquistar e a sustentar vantagem competitiva (Day, 1999; Henderson, 1998; Porter, 1986; 1989; Quinn, 2001); identificam e implementam opções de crescimento adequadas ao contexto competitivo (Ansoff, 1983).

Autores como Whittington (2002), Bowman, Singh e Thomas (2006) e Mintzberg, Ahlstrand e Lampel (2000) construíram tipologias com a intenção de proporcionar uma visão geral sobre as diferentes abordagens sobre a estratégia organizacional. 0 valor dessas classificações está em apontar a ampla variedade de significados que o termo estratégia pode ter no ambiente organizacional. Com base nesses autores, identificamos três grandes leituras ou abordagens: a administrativa, a econômica e a sociocomportamental. As administrativas privilegiam aspectos voltados ao planejamento e aos processos da estratégia. As econômicas enfatizam os recursos, ascapacitações eas vantagens competitivas. E, porfim, as abordagenssociocomportamentais investigam processos psicológicos, sociais e linguísticos relacionados a práticas estratégicas das organizações.

Os estudos comunicacionais podem ser enquadrados nesse último grupo, ao qual é possível relacionar desde os trabalhos de Weick (1995) sobre como os membros da organização constroem sentido em torno de práticas estratégicas, até os trabalhos recentes de autores vinculados à corrente da estratégia como prática (strategy-as-practice), como Hendry (2000), Paali, Vaara e Sorsa (2009) e Seidl (2007), que tratam dos discursos sobre a estratégia. Ainda podemos vincular a esse grupo as pesquisas de autores brasileiros como Bueno (2009), Kunsch (2009), Bulgacov e Marchiori (2010, 2011), Reis, Marchiori e Casali (2010) e Lima (2014; 2015), além da proposta da "nova teoria estratégica" de Pérez (2008, 2012) e de Pérez e Massoni (2009).

Sobre esses antecedentes, construímos esta reflexão, com o objetivo de desvendar elementos comunicacionais para a compreensão da estratégia organizacional. Não estamos, portanto, focalizando a estratégia de comunicação, mas sim a estratégia da organização, que, como já discutimos, é objeto de diferentes abordagens. Partindo da teoria sistêmica, fundamentada em Niklas Luhmann (1997a; 1997b; 2005; 2006; 2011), identificamos na abordagem discursiva de Eliseo Verón (1980; 1996; 2004), uma possibilidade de articulação entre as noções de sistema e de discurso, que é o ponto de partida para nossa discussão.

\section{SISTEMA E DISCURSO}

Conforme Luhmann (2011), os sistemas sociais podem ser compreendidos como unidades operacionalmente fechadas e interativamente abertas, que têm a comunicação como sua operação essencial. É por meio da comunicação que a diferença sistema social/ambiente é (re)produzida continuamente, garantindo a manutenção do sistema e reduzindo a complexidade do ambiente.

A diferenciação dos sistemas sociais parece seguir um movimento reflexivo e recursivo em que o sentido é construído em uma rede de comunicações, que opera de forma circular, autopoiética ${ }^{2}$ e operacionalmente fechada. 0 sistema observa o entorno e constrói sentido tendo por referência esse tecido autorreferenciado de comunicações. Os sentidos circulam nessas redes e constituem o horizonte operativo dos sistemas sociais, os quais, apesar de operacionalmente fechados,

20 adjetivo "autopoiética" é utilizado para designar uma estrutura (sistema) em que ocorre a autopoiese, ou seja, a capacidade de autorreproduzir-se exclusivamente por meio de seus próprios elementos e operações internas. No caso dos sistemas sociais, a comunicação e a rede de comunicações formada no interior do sistema constituem o elemento fundamental para a autorreprodução e são por ele responsáveis (Luhmann, 2011). 
não estão isolados. A linguagem possibilita acoplamentos estruturais com indivíduos e outros sistemas, permitindo que elementos externos aos sistemas sejam incorporados, mantendo, no entanto, a autorreferencialidade na construção dos sentidos. Ou seja, o sistema tem contato com o mundo externo, mas constrói o sentido segundo suas referências internas. Isso garante a identidade, mantendo a diferenciação sistema/ambiente.

Nesse contexto, os discursos são efeitos de sentidos construídos nos sistemas sociais. Podem ser compreendidos também como lances de linguagem ou enunciados que, em jogos de linguagem, materializam os sentidos que circulam nos sistemas. Esses lances parecem estar submetidos a regras e acordos, explícitos ou tácitos, que são intrínsecos à condição de fechamento dos sistemas sociais. Além disso, os lances configuram um contexto social e, com isso, assumem posições, expectativas e disputas.

Com a aproximação entre as noções de sistema e de discurso, chegamos à perspectiva sistêmico-discursiva que fundamenta esta reflexão, contribuindo para direcionar a atenção da investigação para a dimensão social do discurso, não ignorando 0 sujeito, mas deslocando a atenção para a diferença sistema/ambiente e para a dinâmica social da produção de sentidos. Essa perspectiva fornece as noções fundamentais para compreendermos as organizações como sistemas sociais formados por comunicação e os discursos como elementos constituintes desses sistemas, além de indicar um percurso metodológico para uma abordagem discursiva. A partir do tensionamento entre as noções de organização e de comunicação, identificamos um conjunto de elementos comunicacionais para a compreensão da estratégia das organizações. Esses elementos são categorizados da seguinte forma: a decisão, a identificação, a institucionalização, a mediação, a cultura organizacional e a comunicação organizacional.

\section{A decisão}

Com base em Luhmann (2005), partimos do pressuposto de que a decisão é o processo fundamental, que diferencia, define e mantém o sistema organizacional. Ao mesmo tempo em que é responsável pela autorreprodução das organizações, ela possibilita a comunicação direta com outros sistemas. Conforme Rodrigues e Neves (2012), a decisão pode ser exportada para outros sistemas, ou, em outras palavras, pode ser compartilhada com outros sistemas sem prejudicar a autopoiese do sistema organizacional.

As decisões, para Luhmann, constituem, antes de uma operação mental, uma forma específica de comunicação. Nesse sentido, elas não são tomadas e depois comunicadas. As decisões são comunicação. Como as decisões são constituídas por duas distinções entrelaçadas, uma decisão envolve a comunicação, explícita ou implícita, de uma alternativa selecionada e de alternativas rejeitadas. Assim, a comunicação de uma decisão é paradoxal. Ela informa sobre a decisão, mas também sobre as alternativas, que, reitere-se, são e não são alternativas; caso contrário não haveria decisão. Por ser paradoxal, a comunicação da decisão é frágil e demanda uma desconstrução por meio de comunicações posteriores. Uma decisão é conectada a outra em uma rede, de modo que as incertezas sobre o contexto da primeira são reduzidas. As decisões reduzem a complexidade de decisões posteriores, servindo como ponto de referência para estas.

A noção de decisão de Luhmann (2005) é abrangente e parece não se restringir a comportamentos regidos por uma ideia de racionalidade absoluta. Compreender a decisão como uma forma de comunicação pressupõe considerar a racionalidade múltipla envolvida nos comportamentos decisórios. Assim, a decisão deixa de ser um processo sequencial, linear, consciente, deliberado e planejado, em que um conjunto de alternativas é avaliado e a melhor escolha é feita, passando a ser compreendida como a expressão de um conjunto amplo de fatores inter-relacionados que, além da razão, envolvem 
afeto, intuição e relações sociais. Dessa forma, o processo decisório pode ser compreendido como não linear e transitório. Essas características também são ressaltadas por abordagens que procuram integrar os aspectos cognitivos e afetivos do processo decisório, como as de Simon (1970) e de Leitão (1997).

\section{A identificação}

Conforme Luhmann (2011), os sistemas organizacionais, de forma autorreferente, processam sua distinção quanto ao ambiente complexo e, com isso, identificam-se e criam uma identidade que os diferencia. Para o autor (Luhmann, 2011, p. 81), "o sistema é a diferença resultante da diferença entre sistema e meio". Para existirem, os sistemas precisam definir o que os constitui e o que é ambiente, ou seja, o que está fora. Essa diferença é a marca da existência dos sistemas, e ela é construída por meio da autopoiese, com operações internas e exclusivas dos sistemas. Os sistemas se constituem mediante esforços permanentes de distinção entre o que eles são e o que é o ambiente. Portanto, parece possível dizer que eles estão em permanente estado de identificação ou de demarcação de suas fronteiras. Esse estado demanda uma capacidade de auto-observação, em que os sistemas monitoram o ambiente permanentemente e mantêm suas distinções em relação a ele.

0 preceito da diferença parece ser fundamental para a discussão em torno da identificação. Para Woodward (2009), a identidade é relacional, porque, para existir, necessita de algo fora dela, de algo que ela não é. Para Silva (2009), a afirmação da identidade envolve a demarcação de fronteiras, estabelecendo distinções entre o que fica dentro e o que fica fora. Essa diferença é o que marca a identidade e fornece as condições para que ela exista. Assim, é possível dizer que a identidade depende da diferença. Por outro lado, afirmações sobre a diferença, muitas vezes, dependem de uma cadeia, em geral oculta, de declarações negativas sobre outras identidades. Portanto, identidade e diferença constituem noções inseparáveis tanto em uma perspectiva dos estudos culturais (Hall, 2009; Silva, 2009; Woodward, 2009), como em uma perspectiva sistêmica (Luhmann, 2011). Esse paradoxo entre o que é e o que não é, ou, em outras palavras, entre o que faz e o que não faz parte do sistema, está na origem da diferenciação sistêmica.

Em relação às identidades das organizações, compreendemos que elas são construídas sobre essas representações, demarcando as fronteiras simbólicas e sistêmicas, distinguindo o que faz parte dos sistemas e o que é ambiente para as organizações. Fechadas operacionalmente, as organizações procuram construir e manter suas distinções por meio da comunicação. No entanto, a instabilidade, a flexibilidade, a ambiguidade e a contingência também parecem características das identidades organizacionais. No contexto atual, de crescentes pressões ambientais e de novas configurações sociais e organizacionais, em que predominam as descontinuidades (Gomes, 2011), indivíduos e organizações constroem a cada instante suas identidades. A transitoriedade e a flexibilidade, demandadas por ambientes em constante transformação, parecem exigir de indivíduos e de organizações uma multiplicidade de projetos e de identificações ao longo de sua existência. Teríamos, assim, um "feixe de pequenas identificações" (lasbeck, 2009, p. 16), compondo um mosaico, construído a partir das diferenças suscitadas em interações.

Baldissera (2007) defende que as identidades, devido a forças diversas e multidirecionais, são (re)tecidas permanentemente em processos de comunicação. 0 autor propõe a noção de "complexus de identificações", destacando a diversidade de possibilidades identificatórias para indivíduos e organizações. Essas possibilidades configuram uma identidade que se atualiza constantemente, permitindo a manifestação de múltiplas identificações, mas mantendo uma coesão. A identidade, portanto, é fechada e aberta. Nas palavras de Baldissera (2007, p. 231), a organização "fecha-se estrategicamente para construir-se e instituir-se como uma dada coerência/ordenação identitária frente ao outro (sua alteridade), e abre-se para, em tensões/disputas com sua alteridade (de qualquer qualidade)". 


\section{A institucionalização}

Em seus processos identificatórios, as organizações recorrem a práticas, decisões e estruturas legitimadas nos ambientes institucionais. Conforme Vizer (2011, p. 103), o termo instituição define "um conjunto organizativo-simbólico, uma unidade de práxis e sentido". Essa harmonia parece romper-se apenas em situações de crise aguda e de sobrevivência da comunidade. Para o autor, modelos de apropriação social, que atraem e recrutam, por tradição, convicção ou interesse, são produzidos mediante um conjunto de ideias, crenças e práticas legitimadas como instrumentos de poder e de controle. Esse poder de convocação é que seria responsável pela produção e reprodução das estruturas organizativas das instituições.

A instituição pode ser compreendida como um conjunto tanto real-social como simbólico e imaginário que se define como um universo de sentido e de ação social, como uma trama que é constantemente (re)tecida pelos sistemas sociais. Assim, com base na teoria dos sistemas (Luhmann, 2011), parece possível dizer que as instituições são constituídas, de forma autorreferencial, pelos sistemas sociais, em redes de sentidos que são produzidas nos movimentos de autorreprodução dos sistemas sociais e também nas relações entre organizações.

Hasse (2005) lembra que, para a teoria dos sistemas de Luhmann, a sociedade moderna é sustentada por organizações formais. Estruturalmente ligadas a sistemas funcionais, estas incorporam códigos e programas desses sistemas. Isso faz com que haja uma identificação entre organizações e sistemas funcionais. Essa identificação, no entanto, não é fixa, e as organizações até podem funcionar como instâncias de mediação entre sistemas funcionais. Outro aspecto significativo na relação com o ambiente é a possibilidade de comunicação entre organizações, admitida pela teoria sistêmica.

Assim, compreendemos a institucionalização como um processo em que grupos sociais são criados e perpetuados, em uma lógica de dominação ou poder compartilhado, que envolve a busca por legitimação de práticas, decisões e estruturas. Esse processo revela o poder de convocação de um universo autorreferenciado de sentidos, que leva as organizações a (re) produzirem suas próprias estruturas e as estruturas dos sistemas com os quais se relacionam.

\section{A mediação}

Recorrendo a Luhmann (2011), é possível pensar na mediação da linguagem, que permite o acoplamento entre sistemas sociais e consciências (indivíduos). A linguagem disponibiliza o acesso a redes de sentidos, constituídas tanto internamente quanto na interação entre sistemas. Também é possível pensar em mediação quando consideramos a teoria do discurso de Verón (1980). Essas mediações parecem ocorrer no espaço de defasagem e não linearidade entre produção e reconhecimento, responsável por gerar um campo de efeitos e a multiplicação dos sentidos postos em circulação.

Conforme Martín-Barbero (2006), a mediação pode ser pensada como um espaço de constituição da sociedade e não, simplesmente, de transmissão, de veiculação ou de tradução de representações existentes. A partir das ideias do autor, parece possível pensar na mediação como espaços ou instâncias de negociação de sentido, que interferem e alteram os processos de recepção ou reconhecimento. Dessa forma, Martin-Barbero sugere que o olhar comunicacional pressupõe a colocação em primeiro plano dos ingredientes simbólicos e imaginários. A mediação, portanto, envolve as construções culturais e simbólicas, as apropriações e recodificações das mensagens/discursos, que são responsáveis pela criação de sentidos. Ela ocorre mediante as práticas cotidianas, as técnicas incorporadas nos processos organizacionais, os rituais e a circulação de sentidos nas organizações. 
Quando consideramos a mediação na perspectiva sistêmico-discursiva, portanto, não estamos nos referindoà intermediação, filtragem ou intervenção no processo comunicativo, que são sentidos geralmente atribuídos ao termo, conforme lembra Signates (2003). Principalmente no que diz respeito à relação entre organização e comunicação, não nos referimos à mediação em uma perspectiva funcionalista, como forma de facilitação da transmissão de mensagens. Para nós, a mediação é relacionada às dinâmicas de circulação, de multiplicação e de disputa de sentidos tanto nas operações de diferenciação do sistema organizacional quanto nas interações deste com outros sistemas (ambiente). Consideramos, assim como Hasse (2005), que os sistemas organizacionais podem constituir-se em instâncias mediadoras da institucionalização de práticas, procedimentos, regras e conceitos. Dessa forma, a mediação pode ser um elemento processual estreitamente relacionado com a institucionalização e, como veremos a seguir, com a cultura organizacional.

\section{A cultura organizacional}

Conforme Gershon (2005), na perspectiva sistêmica de Luhmann, a cultura é compreendida como uma forma de abordar os paradoxos que são inerentes aos sistemas. Por si só, ela não pode ser considerada um sistema, pois as operações que diferenciam culturas não são as mesmas que distinguem sistemas. Ela pode ser subdividida em várias outras subculturas, sem haver um critério ou operação fundamental para essas divisões. Além disso, diferentemente dos sistemas sociais, as fronteiras entre culturas não podem ser claramente observadas. Uma cultura é considerada como um modo de vida compartilhado em um contexto particular, mas que pode admitir uma infinidade de subculturas, ou seja, alternativas que, não necessariamente, pressupõem diferenças radicais em relação à cultura principal.

Assim, a cultura parece um veículo ou processo para expressar distinções, mas não para produzi-las. Por meio da noção de cultura, os paradoxos inerentes aos sistemas podem ser observados e discutidos. Com isso, para a teoria sistêmica, a cultura, muitas vezes, é utilizada como uma justificativa para problemas de comunicação, tanto entre indivíduos como entre sistemas sociais, e também como uma forma de explicar comportamentos e processos sociais. Portanto, no âmbito de um sistema social, a tendência parece ser, após dificuldades de comunicação, interpretar eventuais diferenças como problemas culturais. Por outro lado, sob essa concepção, a cultura também parece legitimar as diferenças no interior dos sistemas sociais.

Esse olhar sistêmico e processual sobre a cultura pode ser tensionado mediante a agregação da abordagem discursiva, constituindo o que estamos compreendendo como perspectiva sistêmico-discursiva. Articulada com os processos de identificação, de institucionalização e de mediação, a cultura pode ser associada a uma rede simbólica que é construída tanto no interior dos sistemas quanto na relação entre sistemas (sociais e psicológicos), configurando os sentidos que são mobilizados por meio da linguagem.

Em relação à cultura organizacional, há um aspecto que consideramos necessário abordar quando a articulamos com os processos de identificação, de institucionalização e de mediação. Nesse sentido, a cultura pode ser compreendida como um processo político, em que hábitos, valores, formas de conduta funcionam para regular as interações e direcionar os esforços da organização a um interesse específico. Nos ambientes organizacionais, a cultura e as relações de poder que a engendram parecem influenciar diretamente as decisões e, consequentemente, a própria distinção organização/ambiente. A cultura pode, então, ser considerada uma condição produtiva dos discursos organizacionais e, ao mesmo tempo, um processo organizacional de construção da realidade sistêmica e de compartilhamento das formas de ver, interpretar e compreender a realidade. 


\section{A comunicação organizacional}

De acordo com Deetz (2001), a comunicação organizacional pode ser conceituada de três formas diferentes. A primeira possibilidade é compreendê-la como uma especialidade restrita a um departamento específico. A segunda abordagem considera a comunicação um fenômeno que existe na organização como um todo. A terceira vê a comunicação como uma forma de descrever e explicar a organização, não apenas como um fenômeno que ocorre na organização, isto é, a comunicação é a organização. Esta última opção parece a mais próxima da compreensão sistêmico-discursiva. Com base em Deetz (2001), é possível considerar a comunicação e, portanto, as práticas discursivas como a essência da organização.

A comunicação organizacional é compreendida, a partir de abordagens sistêmicas, complexas e discursivas (Baldissera, 2008; Curvello 2009; Deetz, 2001; Fairhurst e Putman, 2010; Fausto Neto, 2008; Marschack e Grant, 2008), como um processo social de circulação, multiplicação e disputa de sentidos, configurado nos desvios entre produção e reconhecimento, formando uma rede complexa de sentidos, em que uma comunicação é condição para existência da outra. Essa rede de sentidos, continuamente (re)produzida no âmbito dos sistemas organizacionais e nas suas relações com o ambiente (formado por outros sistemas e por indivíduos), é engendrada em desvios, dissensos e indeterminações. Além disso, como sistemas constituídos por comunicação, as organizações parecem ser constantemente observadas na/pela sociedade, 0 que pode deflagrar processos de construção e multiplicação de sentidos, que fogem ao seu controle, mas que contribuem, assim como os processos intencionais, para a configuração da organização nos âmbitos interno e externo.

A organização, portanto, é compreendida como um sistema social produzido e configurado a partir da comunicação organizacional, em um processo de autofortificação, em que suas fronteiras são definidas em processos de identificação. No interior do sistema organizacional, a operação fundamental, responsável por sua autorreprodução, é a comunicação em forma de decisão. Nesse contexto sistêmico organizacional, as decisões são tomadas a partir de efeitos de sentidos. As condições produtivas das decisões são constituídas pela cultura organizacional e pelas interações do sistema com 0 ambiente, que, por sua vez, formam uma rede responsável pela mediação e institucionalização dos sentidos.

\section{CONSIDERAÇÕES FINAIS}

Pela articulação entre os elementos comunicacionais chegamos à noção de rede decisória comunicacional, que pode ser associada a concepções do processo de comunicação (Fausto Neto, 2008; Restreppo, 1995), à concepção de rede de decisões de Luhmann (2005) e também à rede de semiose de Verón $(1980,1996)$. Essa articulação indica que a estratégia organizacional é constituída, negociada e representada, por meio da processualidade da comunicação organizacional, em um conjunto de decisões voltadas para a construção e manutenção das fronteiras sistêmicas. Essas decisões são produzidas, reconhecidas e interconectadas na rede decisória comunicacional. A rede, por sua vez: (re)produz processos de identificação voltados para a demarcação das fronteiras sistêmicas; legitima e institucionaliza, em relações de poder, um repertório de decisões ou efeitos de sentido; medeia os sentidos por meio de apropriações e recodificações; representa e, ao mesmo tempo, constrói a cultura organizacional.

A noção de rede aponta para um conjunto de questões que possibilitam uma leitura comunicacional da estratégia das organizações: 

a) Quais posições/identificações são assumidas/construídas pela organização?
b) Como a rede decisória comunicacional institucionaliza decisões/discursos que configuram a estratégia?
c) Como a estratégia é mediada na rede?
d) Quais as marcas da cultura organizacional presentes nas decisões?
e) Como a estratégia é constituída, negociada e representada por meio da comunicação organizacional?

Essa reflexão nos leva a duas implicações centrais: a possibilidade de acessar as estratégias mediante a comunicação organizacional, representada em discursos formais e informais; e a compreensão de que essas estratégias são constituídas e negociadas em/por processos comunicacionais.

\section{REFERÊNCIAS}

ANSOFF, Igor H. Administração estratégica. São Paulo: Atlas, 1983.

BALDISSERA, Rudimar. Tensões dialógico-recursivas entre a comunicação e a identidade organizacional. Organicom-Revista Brasileira de Comunicação Organizacional e Relações Públicas, São Paulo, ECA-USP, n. 7, p. 230-243, 2007.

BALDISSERA, Rudimar. Por uma compreensão da comunicação organizacional. In: SCROFERNEKER, Cleusa. M. A. (Org.). 0 diálogo possível: comunicação organizacional e paradigma da complexidade. Porto Alegre: Edipucrs, 2008. p. 31-50.

BOWMAN, Edward H.; SINGH, Harbir; THOMAS, Howard. The domain of strategic management: history and evolution. In: PETTIGREW, Andrew; THOMAS, Howard; WHITTINGTON, Richard (Org.). Handbook of strategy and management. Thousand Oaks: Sage, 2006. p. 31-54.

BUENO, Wilson da Costa. Comunicação empresarial: políticas e estratégias. São Paulo: Saraiva, 2009.

BULGACOV, Sérgio; MARCHIORI, Marlene. Estratégia como prática: a construção de uma realidade social em processos de interação organizacional. In: MARCHIORI, Marlene (Org.). Comunicação e organização: reflexões, processos e práticas. São Caetano do Sul, SP: Difusão, 2010. p. 149-166.

Strategy as communicational practice in organizations. In: PRE-CONFERENCE IN STRATEGIC COMMUNICATION - ICA CONFERENCE, 2011, Boston. Strategic communication issues. Washington, DC: International Communication Association, 2011. p. 1-22.

CHANDLER, Alfred D. Strategy and structure: chapters in the history of the industrial enterprise. Cambridge: MIT Press, 1998.

CURVELLO, João José Azevedo. A perspectiva sistêmico-comunicacional das organizações e sua importância para os estudos da comunicação organizacional. In: KUNSCH, Margarida M. Krohling (Org.). Comunicação organizacional. Vol. 1. Histórico, fundamentos e processos. São Paulo: Saraiva, 2009. p. 91-106.

DAY, George S. Manutenção da vantagem competitiva: criação e sustentação das vantagens em ambientes competitivos dinâmicos. In: DAY, George S.; REIBSTEIN David J. (Org.). A dinâmica da estratégia competitiva. Rio de Janeiro: Campus, 1999.

DEETZ, Stanley. Conceptual foundations. In: JABLIN, Frederic M.; PUTNAM, Linda L. (Org.). The new handbook of organizational communication: advances in theory, research and methods. Thousand Oaks: Sage, 2001. p. 3-46. 
FAIRHURST, Gail T.; PUTNAM, Linda. As organizações como construções discursivas. In: MARCHIORI, Marlene (Org.). Comunicação e organização: reflexões, processos e práticas. São Caetano do Sul, SP: Difusão, 2010. p. 103-148.

FAUSTO NETO, Antônio. Comunicação das organizações: da vigilância aos pontos de fuga. In: OLIVEIRA, Ivone de L.; SOARES, Ana Thereza N. Interfaces e tendências da comunicação no contexto das organizações. São Caetano do Sul, SP: Difusão, 2008.

GERSHON, Ilana. Seeing like a system: Luhmann for anthropologists. Anthropological Theory, v. 5, n. 2, p. 99-116, 2005.

GOMES, Victor M. L. R. Uma reflexão sobre a construção de identidades e a comunicação no contexto das organizações em rede. Em Questão, Porto Alegre, v. 17, n. 2, p. 209-224, jul./dez. 2011.

HALL, Stuart. Quem precisa da identidade? In: SILVA, Tomaz Tadeu da (Org.). Identidade e diferença: a perspectiva dos estudos culturais. Petrópolis, RJ: Vozes, 2009.

HASSE, Raimund. Systems theory and new institutionalism: a comparison of two approaches to organization sociology. In: SEIDL, David; BECKER, Kai H. (Org.). Niklas Luhmann and organization studies. Copenhagen: Copenhagen Business School Press, 2005.

HENDERSON, Bruce D. As origens da estratégia. In: MONTGOMERY, Cynthia A.; PORTER, Michael E. (Org.). Estratégia: a busca da vantagem competitiva. Rio de Janeiro: Campus, 1998.

HENDRY, John. Strategic decision making, discourse and strategy as social practice. Journal of Management Studies, v. 37 , n. 7, p. 955-977, 2000.

IASBECK, Luiz Carlos A. Identidade organizacional e a construção dos discursos institucionais. In: KUNSCH, Margarida M. Krohling Kunsch (Org.). Comunicação organizacional. Vol. 1. Histórico, fundamentos e processos. São Paulo: Saraiva, 2009. p. 7-29.

KUNSCH, Margarida M. Krohling. Planejamento estratégico da comunicação. In: KUNSCH, Margarida M. Krohling (Org.). Gestão estratégica da comunicação organizacional e de relações públicas. São Caetano do Sul, SP: Difusão, 2009.

LEITÃO, Sérgio Proença. Para uma nova teoria da decisão organizacional. RAP, Rio de Janeiro, v. 31, n. 2, p. 91-107, mar./abr. 1997.

LIMA, Fábia P. A dimensão comunicacional da estratégia: a estratégia organizacional como prática comunicativa na Secretaria de Estado de Fazenda de Minas Gerais. Tese (Doutorado em Ciências da Comunicação) - Escola de Comunicações e Artes, Universidade de São Paulo, São Paulo, 2014.

. A estratégia como prática de comunicação. In: MARQUES, Ângela; OLIVEIRA, Ivone de Lourdes (Org.). Comunicação organizacional: dimensões epistemológicas e discursivas. Belo Horizonte: FAFICH/UFMG, 2015. p. 135-143.

LUHMANN, Niklas. Por que uma "teoria dos sistemas"? In: NEVES, Clarissa E. B.; SAMIOS, Eva M. B. (orgs.). Niklas Luhmann: a nova teoria dos sistemas. Porto Alegre: UFRGS / Goethe-Institut / ICBA, 1997.

. Novos desenvolvimentos na teoria dos sistemas. In: NEVES, Clarissa E. B.; SAMIOS, Eva M. B. (Org.). Niklas Luhmann: a nova teoria dos sistemas. Porto Alegre: UFRGS / Goethe-Institut / CBA, 1997b.

The paradox of decision making. In: SEIDL, David; BECKER Kai H. (Org.). Niklas Luhmann and organization studies. Copenhagen: Copenhagen Business School Press, 2005. 
. A improbabilidade da comunicação. Lisboa: Vega, 2006.

Introdução à teoria dos sistemas. Petrópolis, RJ: Vozes, 2011.

MARSHAK, Robert J.; GRANT, David. Organizational discourse and new organization development practices. British Journal of Management, v. 19, p. 7-19, 2008.

MARTÍN-BARBERO, Jesús. Dos meios às mediações: comunicação, cultura e hegemonia. Rio de Janeiro: Ed. UFRJ, 2006.

MINTZBERG, Henry; AHLSTRAND, Bruce; LAMPEL, Joseph. Safári de estratégia: um roteiro pela selva do planejamento estratégico. Porto Alegre: Bookman, 2000.

PALLI, Pekka; VAARA, Eero; SORSA, Virpi. Strategy as text and discursive practice: a genre-based approach to strategizing in city administration. Discourse \& Communication, v. 3, n. 3, p. 303-318, 2009.

PÉREZ, Rafael Alberto. Estrategias de comunicación. Barcelona: Ariel, 2008.

. Pensar la estrategia. Buenos Aires: La Crujía, 2012.

PÉREZ, Rafael Alberto; MASSONI, Sandra. Hacia una teoría general de la estrategia. Barcelona: Ariel, 2009.

PORTER, Michael E. Estratégia competitiva. São Paulo: Campus, 1986.

Vantagem competitiva: criando e sustentando um desempenho superior. Rio de Janeiro: Elsevier, 1989.

QUINN, James Brian. Estratégias para mudança. In: MINTZBERG, Henry; QUINN, James Brian (Org.). O processo da estratégia. Porto Alegre: Bookman, 2001.

REIS, Maria do Carmo; MARCHIORI, Marlene; CASALI, Adriana M. A relação comunicação-estratégia no contexto das práticas organizacionais. In: MARCHIORI, Marlene (Org.). Comunicação e organização: reflexões, processos e práticas. São Caetano do Sul, SP: Difusão, 2010. p. 167-187.

RESTREPPO, Mariluz J. Comunicación para la dinámica organizacional. Signo y Pensamiento, n. 26, p. 91-96, 1995.

RODRIGUES, Leo Peixoto; NEVES, Fabrício Monteiro. Niklas Luhmann: a sociedade como sistema. Porto Alegre, RS: Edipucrs, 2012.

SEIDL, David. General strategy concepts and the ecology of strategy discourses: a systemic-discursive perspective. Organization Studies, v. 28, n. 2, p. 197-218, 2007.

SILVA, Tomaz Tadeu da. A produção social da identidade e da diferença. In: SILVA, Tomaz Tadeu da (Org.). Identidade e diferença: a perspectiva dos estudos culturais. Petrópolis, RJ: Vozes, 2009.

SIGNATES, Luiz. Estudo sobre o conceito de mediação e sua validade como categoria de análise para estudos de comunicação. Revista Novos Olhares, v. 1, n. 12, p. 4-19, 2003.

SIMON, Herbert A. Comportamento administrativo: estudo dos processos decisórios nas organizações administrativas. Rio de Janeiro: Fundação Getúlio Vargas, 1970. 
VERÓN, Eliseo. A produção de sentido. São Paulo: Cultrix; Edusp, 1980.

La semiosis social: fragmentos de uma teoria de la discursividad. Barcelona: Gedisa, 1996.

Fragmentos de um tecido. São Leopoldo, RS: Ed. Unisinos, 2004.

VIZER, Eduardo A. A trama (in)visível da vida social: comunicação, sentido e realidade. Porto Alegre, RS: Sulina, 2011.

WEICK, Karl E. Sensemaking in organizations. Thousand Oaks, CA: Sage, 1995.

WHITTINGTON, Richard. O que é estratégia. São Paulo: Thomson Pioneira, 2002.

WOODWARD, Kathryn. Identidade e diferença: uma introdução teórica e conceitual. In: SILVA, Tomaz Tadeu da (Org.). Identidade e diferença: a perspectiva dos estudos culturais. Petrópolis, RJ: Vozes, 2009.

Artigo recebido em 8.9.2015 e aprovado em 3.10.2015. 